\title{
Universiteit
}

Leiden

The Netherlands

\section{Duality between spatial and angular shift in optical reflection}

Aiello, A.; Merano, M.; Woerdman, J.P.

\section{Citation}

Aiello, A., Merano, M., \& Woerdman, J. P. (2009). Duality between spatial and angular shift in optical reflection. Physical Review A, 80, 061801. doi:10.1103/PhysRevA.80.061801

Version: $\quad$ Not Applicable (or Unknown)

License: Leiden University Non-exclusive license

Downloaded from: https://hdl.handle.net/1887/60048

Note: To cite this publication please use the final published version (if applicable). 


\title{
Duality between spatial and angular shift in optical reflection
}

\author{
A. Aiello, ${ }^{1,2, *}$ M. Merano, ${ }^{2}$ and J. P. Woerdman ${ }^{2}$ \\ ${ }^{1}$ Max Planck Institute for the Science of Light, Günter-Scharowsky-Straße 1/Bau 24, 91058 Erlangen, Germany \\ ${ }^{2}$ Huygens Laboratory, Leiden University, P.O. Box 9504, 2300 RA Leiden, The Netherlands
}

(Received 14 September 2009; published 7 December 2009)

\begin{abstract}
We report a unified representation of the spatial and angular Goos-Hänchen and Imbert-Fedorov shifts that occur when a light beam reflects from a plane interface. We thus reveal the dual nature of spatial and angular shifts in optical beam reflection. In the Goos-Hänchen case we show theoretically and experimentally that this unification naturally arises in the context of reflection from a lossy surface (e.g., a metal).
\end{abstract}

DOI: 10.1103/PhysRevA.80.061801

PACS number(s): 42.79.-e, 41.20.Jb, 42.25.Gy, 78.20.-e

\section{INTRODUCTION}

In the 17th century Newton was the first to surmise that the center of a reflected beam should present a small spatial shift $\Delta$ in the plane of incidence relative to its geometrical optics position [1]. More than two centuries afterward, in 1947 Goos and Hänchen (GH) [2] were able to quantitatively measure such a shift (see [3] for a literature survey since that time). The GH shift is typically in the subwavelength domain; it has become technologically important in recent years since it directly affects the modes of optical waveguides and microcavities $[4,5]$ and has great potential for (bio)sensor applications [6]. Theoretically, the GH shift has been explained at various levels and several generalizations have been discovered [7-9]. Among the latter it was predicted that the axis of the reflected beam should display a small angular deviation $\Theta$ from the law of specular reflection $\theta_{\text {inc }}=\theta_{\text {ref }}[7,10]$. Interestingly, it took about 50 years since the original experiment performed by Goos and Hänchen to actually observe such angular shift in the microwave [11] and the optical [3] regimes. Presently, it is common wisdom that spatial and angular GH shifts are two different phenomena observables in two mutually exclusive regimes: the spatial GH shift occurs in total reflection (reflected intensity =incident intensity) [12], while the angular GH shift appears in partial reflection (reflected intensity $<$ incident intensity) [10].

In this Rapid Communication we show that this separation is artificial. We present a unified description for the spatial and angular GH shifts that will appear as two aspects of a unique beam-propagation phenomenon. We show that such duality between spatial and angular shift is rather general and also applies to the Imbert-Fedorov (IF) effect, which is a shift normal to the plane of incidence [13] that has drawn considerable interest lately [14-16]. Finally, for the GH shift we show that unification naturally arises in the context of reflection from lossy surfaces. For this case we also furnish an experimental demonstration that the spatial and angular shifts occur simultaneously.

Our Rapid Communication is structured as follows: we give first a qualitative picture of the envisaged unification. Then, we furnish a rigorous theoretical analysis of the beam-

\footnotetext{
*andrea.aiello@mpl.mpg.de
}

propagation problem and show that the unified description actually holds for both the GH and the IF shifts. Finally, we demonstrate, both theoretically and experimentally, that for the GH case lossy reflecting surfaces naturally induce unification.

\section{QUALITATIVE PICTURE}

Consider a system consisting of two homogeneous isotropic media of dielectric constants $\varepsilon_{1}$ and $\varepsilon_{2}$ filling the halfspaces $z<0$ and $z \geq 0$, respectively, as shown in Fig. 1. A monochromatic beam of light of wavelength $\lambda_{0}$ and waist $w_{0}$ propagates along the central wave vector $\mathbf{k}_{0}$ in the region $z$ $<0$ before impinging upon the plane interface of equation $z=0$ that separates medium 1 from medium 2. Detailed derivations of the angular and the spatial displacements $\Theta$ and $\Delta$ for this system have already been reported elsewhere $[7-9,12,17-19]$ and will not be repeated here. We merely quote the basic results in the form

$$
\Delta_{\lambda}=\lambda_{0} \operatorname{Im}\left[D_{\lambda}\right], \quad \Theta_{\lambda}=-\left(\theta_{0}^{2} / 2\right) \operatorname{Re}\left[D_{\lambda}\right]
$$

where $\lambda_{0}=\lambda_{0} /(2 \pi)$, and $\theta_{0}=2 \lambda_{0} / w_{0}$ is the angular spread of the incident beam [20]. The expressions above are valid for both the GH and the IF shifts where the coefficient $D_{\lambda}$ is equal to

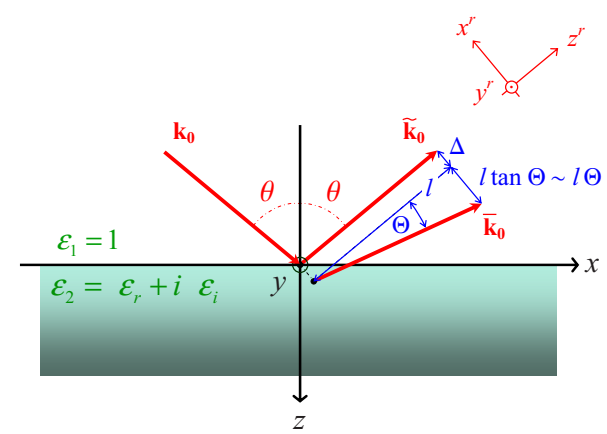

FIG. 1. (Color online) Scheme of the beam reflection at the plane interface. Here $\tilde{\mathbf{k}}_{0}$ and $\overline{\mathbf{k}}_{0}$ are the central wave vectors of the reflected beam as predicted by geometrical and wave optics, respectively. The waist of the incident beam is located at the origin of the laboratory Cartesian frame $x y z$. 


$$
D_{\lambda}=\frac{\partial \ln r_{\lambda}}{\partial \theta}=\frac{1}{R_{\lambda}} \frac{\partial R_{\lambda}}{\partial \theta}+i \frac{\partial \phi_{\lambda}}{\partial \theta} \equiv D_{\lambda}^{\mathrm{R}}+i D_{\lambda}^{\mathrm{I}},
$$

in the GH case, and to $D_{\lambda}=2 i\left[\left(r_{P}+r_{S}\right) / r_{\lambda}\right] \cot \theta$ in the IF case [21]. Here $D_{\lambda}^{\mathrm{R}} \equiv \operatorname{Re}\left[D_{\lambda}\right], D_{\lambda}^{\mathrm{I}} \equiv \operatorname{Im}\left[D_{\lambda}\right]$, and the index $\lambda$ is a label for the two linear polarizations parallel $(\lambda=P$ or TM) and perpendicular $(\lambda=S$ or TE) to the central plane of incidence $x-z$. Moreover, $r_{\lambda} \equiv r_{\lambda}(\theta)=R_{\lambda} \exp \left(i \phi_{\lambda}\right)$ is the Fresnel reflection coefficient [22] evaluated at the central angle of incidence $\theta$, where $R_{\lambda}=\left|r_{\lambda}\right|$ and $\phi_{\lambda}=\arg r_{\lambda}$.

From Fig. 1 it follows, using elementary geometrical considerations, that the total beam displacement observed at distance $l$ from the origin is expressible as a linear combination of $\Delta_{\lambda}$ and $\Theta_{\lambda}$ (supposedly $\Theta_{\lambda} \ll 1$ ) of the form

$$
\delta_{\lambda}(l)=\Delta_{\lambda}+l \Theta_{\lambda} .
$$

From now on, in order to avoid trivial repetitions, we will consider explicitly the GH case only. However, all our conclusions for the $\mathrm{GH}$ case can be straightforwardly extended to the IF case with minimum effort.

Equation (3) supports the hypothesis that $\Delta_{\lambda}$ and $\Theta_{\lambda}$ are two different manifestations of a unique phenomenon, as they can be connected by such an elementary geometric relation. From Eq. (2) it follows that in the case of total reflection $\left(R_{\lambda}=1\right)$, the reflection coefficients reduce to a pure phase factor $r_{\lambda}=\exp \left(i \phi_{\lambda}\right)$, thus, $D_{\lambda}^{\mathrm{R}}=0$ and the reflected beam undergoes a purely spatial shift $\Delta_{\lambda}$. Vice versa, if the reflection coefficients are strictly real, as in the case of airto-glass partial reflection, then $\phi_{\lambda} \in\{0, \pm \pi\} \Rightarrow D_{\lambda}^{\mathrm{I}}=0$, and the beam undergoes a purely angular deflection $\Theta_{\lambda}$. However, when the reflection coefficients are complex, then both $\Delta_{\lambda} \neq 0$ and $\Theta_{\lambda} \neq 0$, and a unified description of spatial and angular GH shifts becomes mandatory. Since for a plane interface between two lossless media the Fresnel coefficients are either purely real or pure phase factors [22], then we have either $\delta_{\lambda}=l \Theta_{\lambda}$ or $\delta_{\lambda}=\Delta_{\lambda}$, respectively, and spatial and angular GH shifts are mutually exclusive. On the other hand, when either one or both of the media are lossy, the separation between spatial and angular GH shifts becomes artificial, and unification naturally occurs.

\section{THEORETICAL DESCRIPTION}

As a first step toward unification, in this section we aim to deduce Eq. (3) from a perfectly general $a b$ initio calculation, without restrictions on the media forming the interface or on the transverse shape of the light beam. It is well known that under minimal hypotheses $[9,20]$ it is possible to obtain a valid angular spectrum representation for the electric field of the reflected beam of the form $\mathbf{A}(\mathbf{X}, Z)=(2 \pi)^{-1} \iint \mathbf{A}(\boldsymbol{\kappa}, Z) e^{i \boldsymbol{\kappa} \cdot \mathbf{X}} d^{2} \boldsymbol{\kappa}$, where $\mathbf{A}(\boldsymbol{\kappa}, Z)$ is uniquely determined by the angular spectrum of the incident beam and the Fresnel reflection coefficients [19]. A Cartesian reference frame attached to the reflected beam with a scaled coordinate system is utilized in which $X=k_{0} x^{r}, \quad Y=k_{0} y^{r}, Z$ $=k_{0} z^{r}$, and $k_{0}=\left|\mathbf{k}_{0}\right|$, as shown in Fig. 1. Moreover, we have defined $\mathbf{X} \equiv X \hat{\boldsymbol{x}}^{r}+Y \hat{\boldsymbol{y}}^{r}$, and $\boldsymbol{\kappa}=\mathbf{k}-\hat{z}^{r}\left(\hat{z}^{r} \cdot \mathbf{k}\right) \equiv \kappa_{1} \hat{\boldsymbol{x}}^{r}+\kappa_{2} \hat{\boldsymbol{y}}^{r}$ is the transverse part of the unit wave vector $\hat{\mathbf{k}} \equiv \mathbf{k} / k_{0}=\boldsymbol{\kappa}+\kappa_{3} \hat{z}^{r} \quad$ with $\quad$ respect to $\hat{z}^{r} \equiv \tilde{\mathbf{k}}_{0} / k_{0}$, with $\kappa_{3}=(1-\boldsymbol{\kappa} \cdot \boldsymbol{\kappa})^{1 / 2}$. Here $\tilde{\mathbf{k}}_{0}=\mathbf{k}_{0}-2 \hat{z}\left(\hat{z} \cdot \mathbf{k}_{0}\right)$ is the central wave vector of the reflected beam as ruled by geometrical optics. The angular spectrum in the plane $Z$ is determined by its value at $Z=0$ via the relation [20]

$$
\mathbf{A}(\boldsymbol{\kappa}, Z)=\mathbf{A}(\boldsymbol{\kappa}, 0) \exp (-i \mathcal{H} Z),
$$

where $\mathcal{H}=-\kappa_{3}$ is the so-called optical Hamiltonian that governs the well-known Hamilton equations of motion for light rays in vacuum [23]:

$$
\frac{d \boldsymbol{\kappa}}{d Z}=-\frac{\partial \mathcal{H}}{\partial \mathbf{X}}=\mathbf{0}, \quad \frac{d \mathbf{X}}{d Z}=\frac{\partial \mathcal{H}}{\partial \boldsymbol{\kappa}}=\frac{\boldsymbol{\kappa}}{\kappa_{3}} .
$$

The analogy between Eq. (4) and the expression for the time evolution of the wave function of a quantum system in the Schrödinger picture suggests the use of an enlightening quantumlike notation [24-26] by writing $\mathbf{A}(\boldsymbol{\kappa}, Z)=\langle\boldsymbol{\kappa} \mid \mathbf{A}(Z)\rangle \quad$ and $\quad \mathbf{A}(\mathbf{X}, Z)=\langle\mathbf{X} \mid \mathbf{A}(Z)\rangle, \quad$ where $|\boldsymbol{\kappa}\rangle=\left|\kappa_{1}, \kappa_{2}\right\rangle$ and $|\mathbf{X}\rangle=|X, Y\rangle$ are the basis vectors in the transverse momentum and position space, respectively. In Eq. (4) the longitudinal coordinate $Z$ has the role of a dimensionless time, then we can write $|\mathbf{A}(Z)\rangle=\exp (-i \hat{\mathcal{H}} Z)|\mathbf{A}(0)\rangle$, where $\hat{\mathcal{H}}$ is the Hamiltonian operator defined via $\left\langle\boldsymbol{\kappa}|\hat{\mathcal{H}}| \boldsymbol{\kappa}^{\prime}\right\rangle=-(1-\boldsymbol{\kappa} \cdot \boldsymbol{\kappa})^{1 / 2} \delta\left(\boldsymbol{\kappa}-\boldsymbol{\kappa}^{\prime}\right)$. In quantum mechanics unitary evolution implies that probabilities are conserved along with propagation, namely, $\langle\mathbf{A}(Z) \mid \mathbf{A}(Z)\rangle=\langle\mathbf{A}(0) \mid \mathbf{A}(0)\rangle$. In our case, this means that the flux of the electric-field energy density across any plane $Z=$ const is independent from $Z$, namely, $\iint|\mathbf{A}(\mathbf{X}, Z)|^{2} d X d Y=\iint|\mathbf{A}(\kappa, 0)|^{2} d^{2} \kappa=1$, where we have renormalized the field amplitude of the reflected beam to ensure $\langle\mathbf{A}(0) \mid \mathbf{A}(0)\rangle=1$. At any given coordinate $Z$ the electric-field energy density $|\mathbf{A}(\mathbf{X}, Z)|^{2}$ gives the spatial beam profile in the observation plane $X-Y$. The $Z$-dependent centroid of such energy distribution $\langle\mathbf{X}\rangle(Z)=\iint \mathbf{X}|\mathbf{A}(\mathbf{X}, Z)|^{2} d X d Y$ measures the deviation of the beam axis with respect to the central axis $\hat{z}^{r}[7,17]$ defined by geometrical optics. If we define the transverse position and momentum operators such that $\hat{\mathbf{X}}\left|\mathbf{X}^{\prime}\right\rangle=\mathbf{X}^{\prime}\left|\mathbf{X}^{\prime}\right\rangle$ and $\hat{\mathbf{K}}\left|\boldsymbol{\kappa}^{\prime}\right\rangle=\boldsymbol{\kappa}^{\prime}\left|\boldsymbol{\kappa}^{\prime}\right\rangle$, respectively, then the centroid of the beam can be evaluated as $\langle\mathbf{X}\rangle(Z)=\langle\mathbf{A}(Z)|\hat{\mathbf{X}}| \mathbf{A}(Z)\rangle$ $=\left\langle\mathbf{A}(0)\left|e^{i \hat{\mathcal{H}} Z} \hat{\mathbf{X}} e^{-i \hat{\mathcal{H}} Z}\right| \mathbf{A}(0)\right\rangle=\left\langle\mathbf{A}(0)\left|\hat{\mathbf{X}}_{H}(Z)\right| \mathbf{A}(0)\right\rangle$, where $\hat{\mathbf{X}}_{H}(Z)$ is the position operator in the Heisenberg picture:

$$
\left\langle\boldsymbol{\kappa}\left|\hat{\mathbf{X}}_{H}(Z)\right| \boldsymbol{\kappa}^{\prime}\right\rangle=\left(-\frac{1}{i} \frac{\partial}{\partial \boldsymbol{\kappa}}+Z \frac{\boldsymbol{\kappa}}{\kappa_{3}}\right) \delta\left(\boldsymbol{\kappa}-\boldsymbol{\kappa}^{\prime}\right) .
$$

The first term on the right side of Eq. (6) coincides with the momentum representation of the $Z$-independent position operator $\hat{\mathbf{X}}$ in the Schrödinger picture [27]. Therefore, from the very definition of $\langle\mathbf{X}\rangle(Z)$, it follows that the expectation value $\langle\mathbf{A}(0)|\hat{\mathbf{X}}| \mathbf{A}(0)\rangle=\langle\mathbf{X}\rangle(0)$ gives both the $\mathrm{GH}$ and IF spatial shifts $\left\langle\hat{X}_{H}(0)\right\rangle$ and $\left\langle\hat{Y}_{H}(0)\right\rangle$, respectively. The second term, which is linear in $Z$, is proportional to the right side of the second equation in Eq. (5) which determines the direction of propagation of classical rays of light, since 
$\boldsymbol{\kappa} / \boldsymbol{\kappa}_{3}=\tan \boldsymbol{\vartheta}_{1} \hat{\boldsymbol{x}}^{r}+\tan \boldsymbol{\vartheta}_{2} \hat{\boldsymbol{y}}^{r}$. As we expect small angular deviations from geometrical optics predictions, we can write $\tan \vartheta_{i} \approx \vartheta_{i}(i=1,2)$, and the exact relation between wave and geometrical optics established by Eqs. (4) and (5) allows us to identify $\partial\left\langle\hat{X}_{H}(Z)\right\rangle / \partial Z \approx \vartheta_{1}$ with the angular GH shift and $\partial\left\langle\hat{Y}_{H}(Z)\right\rangle / \partial Z \approx \vartheta_{2}$ with the angular IF shift. Such identification becomes even more clear by noting that from $[\hat{\mathbf{K}}, \hat{\mathcal{H}}]=0$ and Eq. (6) it follows that

$$
\langle\mathbf{X}\rangle(Z)=\langle\hat{\mathbf{X}}\rangle+Z\left\langle\hat{\mathbf{K}}(1-\hat{\mathbf{K}} \cdot \hat{\mathbf{K}})^{-1 / 2}\right\rangle
$$

which reduces to $\langle\mathbf{X}\rangle(Z) \approx\langle\hat{\mathbf{K}}\rangle+Z\langle\hat{\mathbf{K}}\rangle$ for $\vartheta_{i} \ll 1[28]$, and the angular brackets indicate expectation values with respect to the state $|\mathbf{A}(0)\rangle$. Equation (7) has the same form $\langle\mathbf{X}\rangle(Z)=\boldsymbol{\Delta}+Z \boldsymbol{\Theta}$ as Eq. (3), where $\boldsymbol{\Delta}$ depends on the position operator $\hat{\mathbf{X}}$, and $\boldsymbol{\Theta}$ on the momentum operator $\hat{\mathbf{K}}$ solely, thus, defining unambiguously both a spatial and an angular vector shift of the beam equal to $\boldsymbol{\Delta}=\langle\mathbf{X}\rangle(0)$ and $\boldsymbol{\Theta}=\partial\langle\mathbf{X}\rangle(Z) / \partial Z$, respectively. Note that the dependence of Eq. (7) on the characteristics of the reflecting surface and on the polarization of the incident beam is contained in the form of the state $|\mathbf{A}(0)\rangle$.

Equation (7) establishes the first part of the announced unification by reproducing Eq. (3) that was naively deduced on the grounds of simple geometric reasoning. We emphasize that, because of its vector form, Eq. (7) describes both GH and IF shifts. The next step is to demonstrate that reflection from lossy surfaces induces simultaneously both spatial and angular GH shifts.

\section{LOSS-INDUCED UNIFICATION}

Consider again the system shown in Fig. 1. Assuming air as medium 1, we can write the dielectric constant of medium 2 as $\varepsilon_{2}=\varepsilon_{r}+i \varepsilon_{i}$. Then, we can distinguish that medium 2 is a dielectric, namely, $\varepsilon_{r}>1$, or a metal with $\varepsilon_{r}<0$. For both cases, from Eq. (2) and the well-known expressions for the Fresnel reflection coefficients [22], it follows that

$$
\begin{gathered}
D_{P}=\frac{2 \sin \theta}{\sqrt{\left(\varepsilon_{r}-\sin ^{2} \theta\right)+i \varepsilon_{i}}} \\
\times \frac{\varepsilon_{i}^{2}+\varepsilon_{r}\left(1-\varepsilon_{r}\right)+i \varepsilon_{i}\left(1-2 \varepsilon_{r}\right)}{\left(\varepsilon_{r}-\sin ^{2} \theta\right)+\left(\varepsilon_{i}^{2}-\varepsilon_{r}^{2}\right) \cos ^{2} \theta+i \varepsilon_{i}\left(1-2 \varepsilon_{r} \cos ^{2} \theta\right)}, \\
D_{S}=\frac{2 \sin \theta}{\sqrt{\left(\varepsilon_{r}-\sin ^{2} \theta\right)+i \varepsilon_{i}}} .
\end{gathered}
$$

To elucidate the role of the losses, we expand Eq. (8) in a Taylor series around $\varepsilon_{i}=0$ and keep terms up to the first order in $\varepsilon_{i}$. For the sake of simplicity, from now on we will consider only the metal case for which the Taylor expansion furnishes

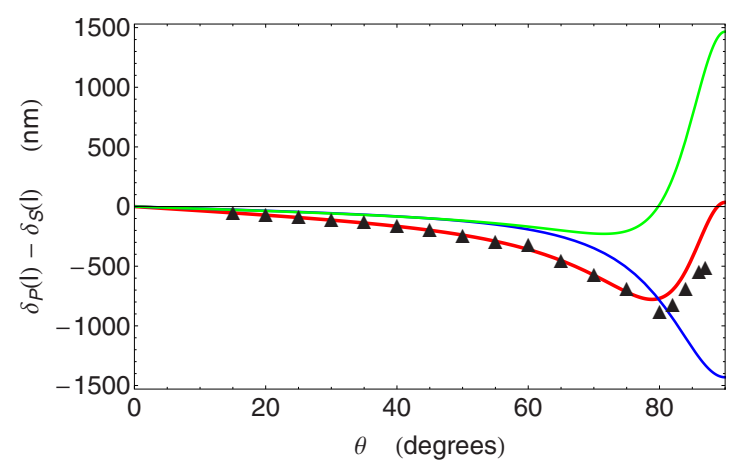

FIG. 2. (Color online) $\delta_{P}(l)-\delta_{S}(l)$ at $l=11.9 \mathrm{~cm}$ for a fundamental Gaussian beam with wavelength $\lambda_{0}=820 \mathrm{~nm}$, waist $w_{0}=32 \mu \mathrm{m}$, incident on a gold surface with $\varepsilon_{2}=-29.02+i 2.03$. Lower (blue) line, $\Delta_{P}-\Delta_{S}$; upper (green) line, $l\left(\Theta_{P}-\Theta_{S}\right)$; central (red) line, $\delta_{P}-\delta_{S}$; black triangles, measured $\delta_{P}-\delta_{S}$.

$$
\begin{gathered}
D_{P}^{\mathrm{R}}=\varepsilon_{i} \frac{\sin \theta\left(1-\varepsilon_{r}\right)^{2}}{\left(\sin ^{2} \theta-\varepsilon_{r}\right)^{3 / 2}} \frac{2 \sin ^{4} \theta-\varepsilon_{r}\left(\sin ^{2} \theta+\varepsilon_{r} \cos ^{2} \theta\right)}{\left(\sin ^{2} \theta-\varepsilon_{r}\right)+\varepsilon_{r}^{2} \cos ^{2} \theta}, \\
D_{P}^{\mathrm{I}}=\frac{-2 \varepsilon_{r} \sin \theta}{\sqrt{\sin ^{2} \theta-\varepsilon_{r}}\left(\sin ^{2} \theta-\varepsilon_{r} \cos ^{2} \theta\right)},
\end{gathered}
$$

for $P$ polarization, and $D_{S}^{\mathrm{R}}=\varepsilon_{i} 2 \sin \theta /\left(\sin ^{2} \theta-\varepsilon_{r}\right)^{3 / 2}$, $D_{S}^{\mathrm{I}}=-2 \sin \theta / \sqrt{\sin ^{2} \theta-\varepsilon_{r}}$, for $S$ polarization. From the expressions above we can see that for an ideal lossless metal $\left(\varepsilon_{i}=0\right)$ we have that $D_{\lambda}$ is purely imaginary and only the spatial shift occurs [29]. However, when losses are "turned on" by letting $0<\varepsilon_{i} \ll 1$, a first-order real part must be added to $D_{\lambda}^{\mathrm{I}}$ causing the simultaneous existence of both spatial and angular shifts. Such loss-induced coexistence between $\Delta$ and $\Theta$ is clearly illustrated in Fig. 2 where Eq. (3) is displayed for reflection at an air-gold interface. The central (red) curve represents the total beam shift at distance $l$ from the origin and it is given, according to Eq. (3), by the sum of the upper (green) curve (angular shift) and the lower (blue) one (spatial shift). This completes the second part of our unification program.

\section{EXPERIMENT}

The experimental setup is sketched in Fig. 3. The $820 \mathrm{~nm}$ output of a superluminescent light-emitting diode (SLED) is spatially filtered by a single-mode optical fiber (SMF) to

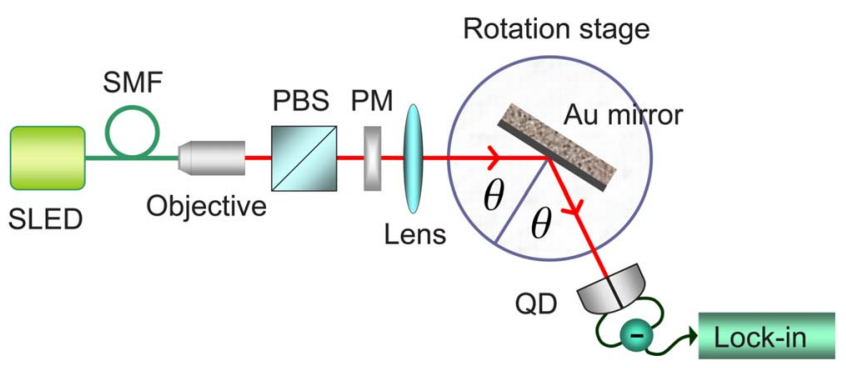

FIG. 3. (Color online) Layout of the experimental setup. 
select a TEM $_{00}$ mode. This is then collimated by a microscope objective and sent through a Glan polarizing prism (PBS) to fix its polarization to $P$. A polarization modulator (PM) switches the beam polarization between $P$ and $S$ to exploit the fact that both spatial and angular $\mathrm{GH}$ shifts are polarization dependent. Then, a lens is used to focus the beam to the desired spot size in front of the mirror. While the spatial GH shift is unaffected by such beam focusing, the angular GH shift depends on the beam angular aperture. The reason for this behavior is evident from the $\theta_{0}^{2}$ factor in Eq. (1), and its physical origin is explained in [3]. Finally, the difference signal from a quadrant detector (QD) is fed into a lock-in amplifier in order to detect the beam displacement in the plane of incidence when polarization is switched between $S$ and $P$. See $[3,30]$ for further details.
The results of the measurements are shown in Fig. 2 along with theoretical prediction. The good agreement confirms the simultaneous occurrence of the spatial and the angular $\mathrm{GH}$ shifts in the lossy regime.

\section{CONCLUSIONS}

In this Rapid Communication we have presented a unified description for spatial and angular Goos-Hänchen shifts occurring in light beam reflection from lossy surfaces. Such description applies to the Imbert-Fedorov effect as well. The unification theory has been worked out for a weakly absorbing metal and the corresponding GH shift has been observed experimentally in reflection from an air-gold plane interface.
[1] I. Newton, Opticks (Dover, New York, 1952); see query 4 on p. 339.

[2] F. Goos and H. Hänchen, Ann. Phys. 436, 333 (1947).

[3] M. Merano, A. Aiello, M. P. van Exter, and J. P. Woerdman, Nat. Photonics 3, 337 (2009).

[4] D. H. Foster, A. K. Cook, and J. U. Nöckel, Opt. Lett. 32, 1764 (2007).

[5] J. Unterhinninghofen, J. Wiersig, and M. Hentschel, Phys. Rev. E 78, 016201 (2008).

[6] X. Yin and L. Hesselink, Appl. Phys. Lett. 89, 261108 (2006).

[7] M. A. Porras, Opt. Commun. 131, 13 (1996).

[8] W. Nasalski, T. Tamir, and L. Lin, J. Opt. Soc. Am. A 5, 132 (1988).

[9] M. McGuirk and C. K. Carniglia, J. Opt. Soc. Am. 67, 103 (1977).

[10] J. W. Ra, H. L. Bertoni, and L. B. Felsen, SIAM J. Appl. Math. 24, 396 (1973).

[11] D. Müller, D. Tharanga, A. Stahlhofen, and G. Nimtz, Europhys. Lett. 73, 526 (2006).

[12] K. Artmann, Ann. Phys. 437, 87 (1948).

[13] O. C. de Beauregard and C. Imbert, Phys. Rev. Lett. 28, 1211 (1972).

[14] M. Onoda, S. Murakami, and N. Nagaosa, Phys. Rev. Lett. 93, 083901 (2004).

[15] K. Y. Bliokh and Y. P. Bliokh, Phys. Rev. Lett. 96, 073903 (2006).

[16] O. Hosten and P. Kwiat, Science 319, 787 (2008).

[17] A. Aiello and J. P. Woerdman, Opt. Lett. 33, 1437 (2008).
[18] A. Aiello, M. Merano, and J. P. Woerdman, Opt. Lett. 34, 1207 (2009).

[19] A. Aiello and J. P. Woerdman, e-print arXiv:0903.3730.

[20] L. Mandel and E. Wolf, Optical Coherence and Quantum Optics, 1st ed. (Cambridge University Press, Cambridge, UK, 1995).

[21] In this case we denote with $\Delta_{\lambda}$ and $\Theta_{\lambda}$, respectively, the spatial and angular separation between the two right-circularly and left-circularly polarized components of the reflected beam generated by the reflection-induced splitting of the $\lambda$-polarized $(\lambda \in\{P, S\})$ incident beam [13].

[22] M. Born and E. Wolf, Principles of Optics, 7th ed. (Cambridge University Press, Cambridge, UK, 2003).

[23] T. Sekiguchi and K. B. Wolf, Am. J. Phys. 55, 830 (1987).

[24] D. Gloge and D. Marcuse, J. Opt. Soc. Am. 59, 1629 (1969).

[25] D. Stoler, J. Opt. Soc. Am. 71, 334 (1981).

[26] M. V. Berry, J. Mod. Opt. 50, 63 (2003).

[27] All the operators without the subscript " $H$ " must be intended in the Schrödinger picture.

[28] The reader knowledgeable about paraxial optics will easily recognize from Eq. (7) in the limit $\vartheta_{i} \ll 1$, and from the relation $\hat{\mathbf{K}}_{H}(Z)=\hat{\mathbf{K}}$, the free-space propagation $A B C D$-matrix law: $\left(\begin{array}{l}\hat{\mathbf{x}}_{H} \\ \hat{\mathbf{K}}_{H}\end{array}\right)(Z)=\left(\begin{array}{ll}1 & Z \\ 0 & 1\end{array}\right)\left(\begin{array}{l}\hat{\mathbf{X}} \\ \hat{\mathbf{K}}\end{array}\right)$.

[29] M. Merano, A. Aiello, G. W. 't Hooft, M. P. van Exter, E. R. Eliel, and J. P. Woerdman, Opt. Express 15, 15928 (2007).

[30] M. Merano, J. B. Götte, A. Aiello, M. P. van Exter, and J. P. Woerdman, Opt. Express 17, 10864 (2009). 